\title{
First sale for quantum computing
}

\section{But critics say that D-Wave's system is still something of a black box.}

\section{BY ZEEYA MERALI}

$\mathrm{I}$ $t$ could turn out to be a milestone for quantum computing. Last week, D-Wave Systems of Burnaby in British Columbia, Canada, announced the first sale of a commercial quantum computer, to global security firm Lockheed Martin, based in Bethesda, Maryland.

Yet perhaps fittingly for a quantum device, uncertainty persists around how the impressive black monolith known as D-Wave One actually works. Computer scientists have long questioned whether D-Wave's systems truly exploit quantum physics, and although the company last month published a paper in Nature (M. W. Johnson et al. Nature 473, 194-198; 2011) to help verify its quantum credentials, some say that the technique used is still in doubt.

Quantum computers could revolutionize the way we tackle problems that stump even the best classical computers, which store and process their data as 'bits' essentially a series of switches that can be either on or off.

The power of quantum bits - or qubits - is that they can be on and off simultaneously. Connect enough qubits together using quantum entanglement and a computer should be able to zip through a multitude of calculations in parallel, at astonishing speed.

But quantum computers are notoriously difficult to construct, with most research groups struggling to entangle more than a handful of qubits. So D-Wave's claims to have a working 128-qubit processor - combined with the company's former reluctance to publish details of the technique in peer-reviewed journals - have long raised eyebrows, explains Scott Aaronson, a computer scientist at the Massachusetts Institute of Technology in Cambridge.

This did not deter Lockheed Martin, however. Communications manager Thad Madden says that the company spent a year reviewing the $\mathrm{D}$-Wave One computer before purchasing it. Although D-Wave has been cagey about specific applications, Lockheed Martin plans to use the technology to help it build "cyber-physical systems", which integrate software with environmental sensors, says Madden.

D-Wave's co-founder, Geordie Rose, says that the sale demonstrates that quantum computing is finally living up to its decades-long promise. Aaronson, however, thinks that the computer-science community will need more

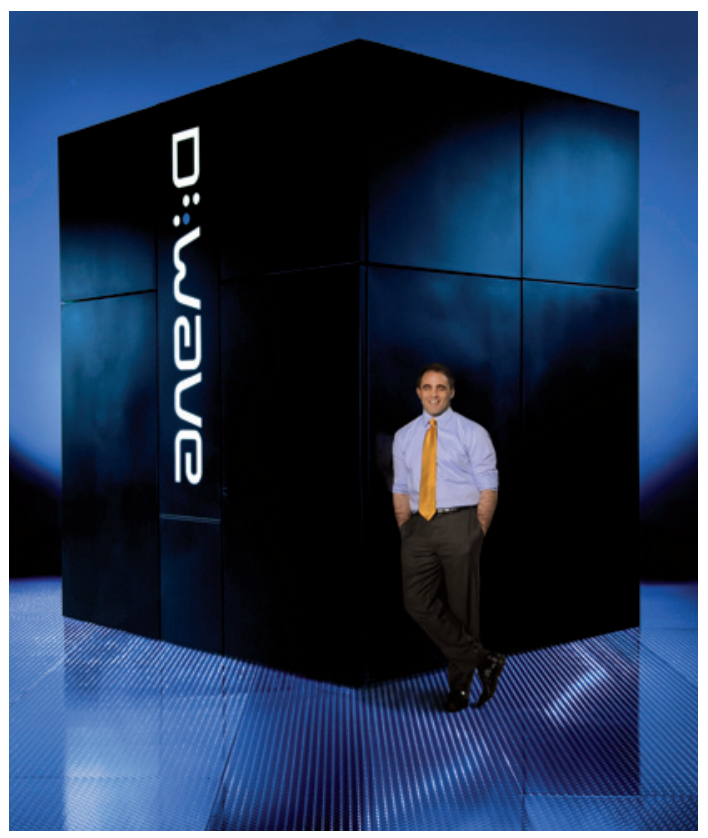

D-Wave co-founder Geordie Rose with his black monolith.

convincing. "Just because a flagship company has bought the system, doesn't mean that it now works," he says.

That mistrust goes back to 2007, when D-Wave apparently demonstrated a 16-qubit computer that could solve a Sudoku puzzle. Many computer scientists and physicists suggested that the device was actually being driven by plain old classical physics. At the time, D-Wave did not respond with any publications ruling out this possibility.

But the company's paper in Nature demonstrated definitive quantum behaviour in a system with eight qubits, made from superconducting niobium loops. Magnetic fields manipulate the combined energy state of the qubits until the system defines the parameters of the problem to be solved. Because the system exists in a quantum superposition, it can simultaneously 'search' through multiple energy states that each represent different solutions, explains Rose. Cooling the system snaps it out of the superposition, so that it settles into a single, low-energy state that represents the final answer, an approach known as quantum annealing (W. D. Oliver Nature 473, 164-165; 2011). D-Wave One uses sixteen of these eightqubit cells.

Sceptics had argued that the system could actually work by using thermal fluctuations - rather than quantum effects - to jostle the qubits through various energy states. However, because these classical effects are significant only above 45 millikelvin, and D-Wave's latest paper shows that its system works below that barrier, only quantum effects can be responsible.

Aaronson says that the paper is a step in the right direction, but only a small one. "There is an enormous gap between demonstrating some kind of quantum effect in eight qubits, as they have done here, and saying that they have a 128-qubit chip that can perform a computationally interesting task faster than a conventional computer," he says. He notes that D-Wave has not demonstrated that the qubits are entangled, a property that most physicists think is essential for quantum computing. "I genuinely hope that D-Wave succeeds," he adds. "But I will only believe it has when I see evidence for it." - SEE FEATURE P.24

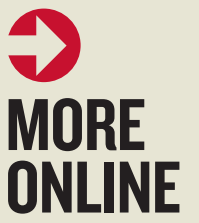

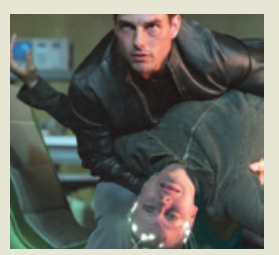

United States field-tests terrorist 'pre-crime' detector go.nature.com/ d4uuwy

\section{OTHER NEWS}

- Smart-REDD plan targets causes of deforestation go.nature.com/udpmwl - Link between biomarkers and disease is often exaggerated go.nature. com/vog9zg

- Roman ship had on-board fish tank go.nature.com/bhhgyk
Q\&A

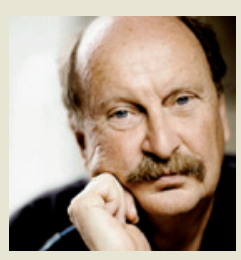

Edzard Ernst, the world's first professor of alternative medicine, is stepping down go.nature.com/ojxiyo 